\title{
Surveilling Non-Opioid Substance Use: Utilizing Multiple Data Sources in Marion County, Indiana
}

\author{
Brittany K. Yarnell, James M. Groh \\ Epidemiology, Marion County Public Health Department, Indianapolis, Indiana, United States \\ Objective
}

To assess the prevalence of non-opioid substance use - including cocaine, methamphetamine and "spice"-within Marion County, Indiana and propose response recommendations utilizing a current opioid response plan.

\section{Introduction}

Cocaine, methamphetamine, and "spice" are addictive, non-opioid substances that negatively impact a person's health through direct and indirect means. Direct health concerns of non-opioid substance use include anxiety, paranoia, seizure, heart attack, stroke, and potentially death while indirect health concerns include the acquisition of disease and infections, particularly sexually transmitted infections (STIs). Substance users experience an increased risk of acquiring STIs since they may exchange sex for substances, use substances within a social setting that may lead to sexual activity, or engage in risky sexual behavior as a result of impaired judgement associated with substance use. The current study evaluated the use of multiple data sources to monitor changes in the rate of cocaine, methamphetamine, and "spice" related emergency department visits as well as cocaine- and methamphetamine-related death rates, within Marion County, Indiana between 2013 and 2017.

\section{Methods}

Two data sources were used in this study. First, prevalence rates of non-opioid substance related emergency department (ED) visits were calculated using Marion County (IN) ED data from Electronic Surveillance System for the Early Notification of Community-Based Epidemics (ESSENCE) between 2013 and 2017. Second, cocaine and methamphetamine death rates were calculated using coroner toxicology data related to Marion County deaths between 2013 and 2017. Cocaine and methamphetamine deaths were defined as any death in which cocaine and methamphetamine was found in the toxicology results, respectively. All rates were calculated per 100,000 and age-adjusted to the 2000 U.S. Census using SAS Enterprise Guide v7.1.

\section{Results}

Non-opioid substance related ED visits have persistently risen between 2013 and 2017 (Figure 1). Methamphetamine and "spice" related ED visits exhibited similar prevalence patterns, increasing from $0.99(0.72,1.58)$ to $5.32(4.67,6.21)$ and 0.46 $(0.28,1.00)$ to $4.13(3.57,4.94)$ per 100,000 , respectively, between 2013 and 2016. Cocaine-related ED visits consistently exhibited the highest prevalence rates, ranging from $3.72(3.17,4.44)$ to $23.56(22.16,25.11)$ per 100,000 in 2013 and 2016 , respectively. In 2017, all non-opioid substance related ED visits drastically increased to 47.78 (45.79, 49.91), 48.48 (46.48, 50.67), and $42.08(40.23,44.13)$ per 100,000 for cocaine, methamphetamine, and "spice," respectively. Further, we looked at cocaine- and methamphetamine-related death rates using coroner toxicology results. We found that between 2013 and 2017, the cocaine-related death rate nearly tripled, from $4.82(4.20,5.64)$ per 100,000 in 2013 to $13.01(11.97,14.23)$ per 100,000 in 2017 (Figure 2). Similarly, methamphetamine-related death rates increased from $1.31(0.99,1.92)$ per 100,000 in 2013 to $10.15(9.25,11.28)$ per 100,000 in 2017 (Figure 2). We did not calculate death rates of those who were found to have "spice" in their system at the time of death due to low prevalence.

\section{Conclusions}

The increase of non-opioid substance related ED visits in Marion County may indicate that non-opioid substance useparticularly cocaine, methamphetamine, and "spice"-may be an emerging public health issue in Marion County. This growing concern is further supported by the consistent increase in cocaine- and methamphetamine-related death rates. A limitation to our study is the inconsistent reporting of the substance in ED chief complaints and missing fields for discharge diagnoses and triage notes. As such, this inconsistency may have led to an underestimation of the prevalence rates of nonopioid substance related ED visits. The addition of triage notes and more reliable discharge diagnoses in 2017 ultimately culminated in a sharp increase in non-opioid substance related ED visits in 2017.

ISDS Annual Conference Proceedings 2019. This is an Open Access article distributed under the terms of the Creative Commons AttributionNoncommercial 3.0 Unported License (http://creativecommons.org/licenses/by-nc/3.0/), permitting all non-commercial use, distribution, and reproduction in any medium, provided the original work is properly cited. 
Certain aspects of Marion County Public Health Department's established opioid response plan may be used to address the growing concern of non-opioid substance use. These aspects include, but are not limited to, engaging community partners, creating a task force, establishing focus groups, and providing resources. While these aspects are critical to effectively respond to non-opioid substance use epidemics, establishing the various components prior to an outbreak enable communities to reduce the impact of such epidemics, if not prevent them from occurring. Additionally, it is important to incorporate participatory aspects into a non-opioid substance response plan such that community members are the driving force to provide context for the impact that non-opioid substance use is having on the community while also offering insight into which interventions would be most effective.
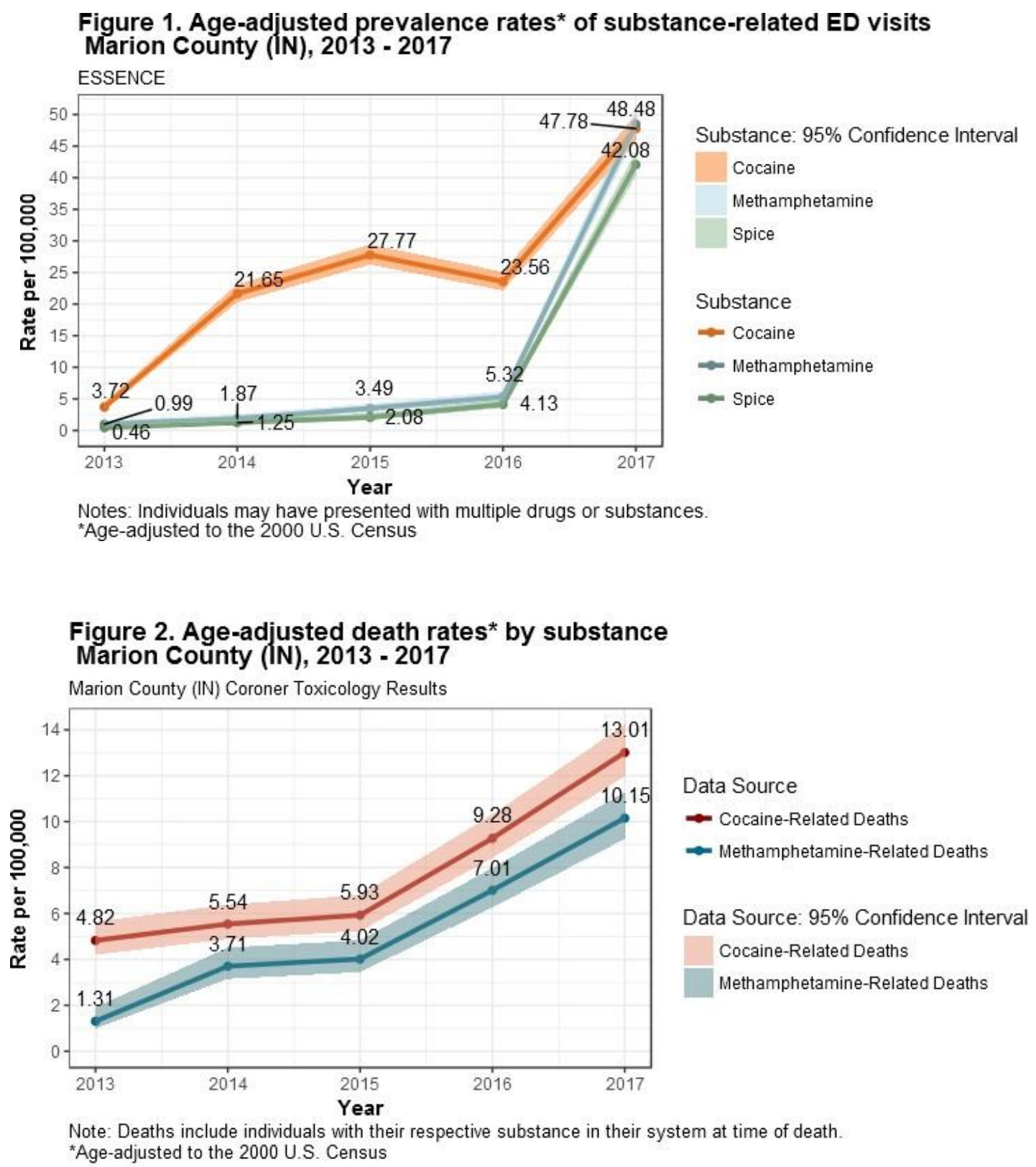